\title{
Using peripheral blood immune signatures to stratify patients with adult and
}

juvenile inflammatory myopathies

Meredyth G LI Wilkinson ${ }^{1,2,3}$, Anna Radziszewska1, Chris Wincup ${ }^{1,4}$, Yiannis loannou ${ }^{1,2}$, David A Isenberg ${ }^{1,2,4}$, Jessica J Manson ${ }^{4}$, Elizabeth $C$ Jury ${ }^{1,2}$

\section{Affiliations:}

1. Division of Medicine, University College London, The Rayne Building, 4th Floor, 5 University Street, London, WC1E 6JF, UK

2. Arthritis Research UK Centre for Adolescent Rheumatology, University College London Hospital and Great Ormond Street Hospital, London, United Kingdom

3. Infection Inflammation and Rheumatology, UCL Great Ormond Street Institute of Child Health, 30 Guilford Street, London, WC1N 1EH

4. Department of Rheumatology, $3^{\text {rd }}$ Floor Central, University College London Hospital, 250 Euston Road, London, NW1 2PG

\section{Contact address:}

Infection Inflammation and Rheumatology,

UCL Great Ormond Street Institute of Child Health,

30 Guilford Street,

London,

WC1N 1EH

Email: meredyth.wilkinson.14@ucl.ac.uk 


\section{ABSTRACT:}

Objective: The inflammatory idiopathic myopathies (IIM) are a group of rare autoimmune diseases defined by muscle weakness and characterised by proinflammatory infiltrates in muscle. Little is known about the immunological profile in peripheral blood of these patients and how this relates to IIM subtypes. This study aimed to stratify adult and juvenile-onset IIM patients according to immune cell profile.

Methods: Peripheral blood mononuclear cells from 44 patients with adult myositis (AM), 15 adolescent-onset juvenile dermatomyositis (a-JDM), and 40 age-matched healthy controls were analysed by flow cytometry to quantify 33 immune cell subsets. AM patients were grouped according to myositis subtype; dermatomyositis (DM) and polymyositis (PM), and also autoantibody specificity. Disease activity was determined by the myositis disease activity assessment tool and clinicians' decision on treatment.

Results: Unique immune signatures were identified for DM, PM and a-JDM compared to healthy controls. DM patients had a T-cell signature comprising increased $\mathrm{CD}^{+}$and $\mathrm{TH} 17$ cell frequencies and increased immune cell expression of IL-6. PM patients had a B-cell signature with reduced memory B-cells. A-JDM had decreased naïve B-cells and increased CD4+T-cells. All patient groups had decreased $\mathrm{CD} 8{ }^{+}$central memory $\mathrm{T}$-cell frequencies. The distinct immune signatures were also seen when AM patients were stratified according to auto-antibody expression; patients with anti-synthetase-antibodies had reduced memory B-cells and patients with autoimmune rheumatic disease overlap had an elevated Th17 profile.

Conclusion: Unique immune signatures were associated with adult versus juvenile disease. The Th17 signature in DM patients supports the potential use of IL-17A inhibitors in treatment of IIMs. 
Keywords: Myositis, immune signature, Th17, interleukin-6

\section{KEY MESSAGES}

- Unique peripheral blood immune signatures identify sub-groups of Inflammatory Idiopathic Myopathies (IIM).

- Adult dermatomyositis (ADM) patients have a Th17 signature and elevated IL6 production.

- Immune stratification could identify adult dermatomyositis (ADM) patients that would benefit from anti-IL-17 or anti-IL-6. 


\section{INTRODUCTION}

The Inflammatory Idiopathic Myopathies (IIM) are a group of rare myopathic autoimmune diseases diagnosed in both adults and children. Patients present with a variety of features including proximal muscle weakness and, in the case of dermatomyositis (DM), skin changes. Up to $80 \%$ of patients have unique serum autoantibody profiles with associated clinical features(1). However, although immunohistochemical analysis of muscle tissue from IIM patients has identified immune cell infiltrate and the expression of pro-inflammatory cytokines(2, 3), very little is known about the peripheral immunological profile in juvenile and adult patient groups(4).

The adaptive immune system is implicated in the pathogenesis of IIM(4) and distinct patterns of immune cell accumulation have been described in muscle and skin biopsies from polymyositis (PM) and DM patients $(5,6)$. The endomysial cellular infiltrates most commonly detected in PM, constitute of CD8+ T cells, CD4+ T cells, dendritic cells (DCs) and macrophages. These cells localise around the muscle fibres. In DM, the perivascular infiltrates consist of CD4+ T cells, DCs, macrophages and B cells. Unlike PM, these cells are found around the inflamed blood vessels (7).

$T$ cells are dysfunctional in the context of IIM. CD4+CD25+FOXP3+ regulatory $T$ cells were shown to lose their ability to regulate the immune system in patients with active JDM $(8,9)$ and pro-inflammatory T helper-17 (Th17) cells have been found in tissue biopsies from IIM patients and were positively correlated with disease activity (7, 9-11). Conversely, B cells have not been thought to be as vital as $T$ cells in the pathogenesis of IIM although they do play an important role. Not only are B cells found in the infiltrates of muscle tissue in DM patients, there is a high detection of myositis-associated (MSA) and specific (MSS) autoantibodies (12-14). The expression of $B$ cell activating factor (BAFF) has been associated with anti-Jo-1 antibodies in DM muscle tissue, specifically in the perifascicular area (15). Recently we have shown that immature $B$ cells are expanded in juvenile dermatomyositis and are skewed towards a pro-inflammatory phenotype by Toll-Like Receptor 7 and Interferon- $\alpha(16)$. 
Most studies on IIM have focused on the inflammatory milieu found within the tissue biopsy. The biopsy is a key diagnostic tool, but is rarely repeated past initial presentation, thus little is known about the histological changes during the disease course. On the other hand little is known about the peripheral immunological profile and how this changes with disease activity. This study investigated the peripheral blood immunological profile from adult and juvenile IIM patients. 


\section{MATERIALS AND METHODS:}

\section{Patients and controls}

Peripheral blood mononuclear cells (PBMC) from 44 adult myositis (AM) patients (including dermatomyositis (DM) and polymyositis (PM)), 15 adolescent-onset juvenile dermatomyositis (a-JDM) patients, 25 age, sex and ethnicity-matched adult healthy controls $(\mathrm{AHC})$, and 15 age, sex and ethnicity-matched teenage healthy controls (THC) were collected over a period of two years and were stored frozen in liquid nitrogen for a maximum time of one year (as previously recorded (16) at the Centre of Adolescent Rheumatology University College London Biobank(16). Participants were recruited after obtaining written informed consent with appropriate ethical approval (REC11/0101). Demographic and clinical information for patients and controls are shown in Table 1. A-JDM patients met Bohan and Peter criteria for probable or definite disease(17). Patients were diagnosed on the basis of their clinical features, serum creatine kinase, electromyogram, magnetic resonance imaging and muscle biopsy results. Myositis-associated and specific autoantibody specificities were recorded when performed as part of routine diagnostic procedure as part of the extended myositis autoantibody panel (Table-2).Disease activity was assessed using the myositis disease activity assessment tool $(\operatorname{MITAX})(18,19)$ and/or the clinicians' treatment decision (increase, decrease or no change in treatment). AJDM patient disease activity was measured by the manual muscle test (MMT8)(20). To calculate the MITAX score; in each of the different organs and systems assessed a patient deemed sufficiently active to require at least $20 \mathrm{mg}$ of steroids $+/$ - an immunosuppressive drug is assessed as a grade $A$ (12 points); a patient with moderately active disease requiring a lesser amount of steroid/immunosuppressive is assessed as a B (8 points); a patient with mild disease requiring topical treatments only or Hydroxychloroquine/non-steroidal anti-inflammatories is assessed as a C (1 point); a patient whose disease was once active in the individual organ system but is no longer active is assessed as a D (0 points); and a patient whose organ systems were never previously involved is assessed as an E (0 points). Patients were categorised into; active, mildly active, remission on-treatment and remission offtreatment. 


\section{Flow cytometry}

PBMCs were analysed by flow cytometry after staining for viability (Median average - AM (95.30\%), AHC (94.35\%), JDM (86.70\%), THC (85.20\%) Supplementary Figure 2 and gating strategy Supplementary Figure 3. Surface staining ex-vivo with directly conjugated antibodies and intracellular cytokines according to previously defined protocols after 4hr culture with PMA, ionomycin and Golgi plug(16) (Supplementary Table 1). Data were collected on an LSRII flow cytometer (BD Pharmingen) using FACS Diva software and analysed by Flowjo (Treestar).

\section{Statistical analysis}

Statistical analysis was performed in GraphPad Prism 6 and SPSS (V.24). Unpaired student $t$-tests were used to compare ex-vivo cell populations from patients and controls. Fold change was calculated to determine the directional change in the mean average. Multiple comparisons were made comparing each cell population using multiple $t$ tests with Holm-Sidak method of correction for multiple comparisons or one-way ANOVA and Tukey's multiple comparison test. MultiExperiment Viewer $(\mathrm{MeV})$ was used to produce heat maps and perform hierarchical clustering. 


\section{RESULTS:}

No significant difference in demographic profile was identified between IIM patient groups

Comparison of the clinical and demographic characteristics of $A M$ and a-JDM patients are shown in Tables 1 and 2. The AM cohort included patients with, DM $(n=19)$, PM $(n=9)$, DM with cancer $(n=4), D M(n=7)$ and PM $(n=5)$ with overlap autoimmune rheumatic diseases (e.g. systemic lupus erythematosus, scleroderma and rheumatoid arthritis).

Myositis-associated and myositis-specific autoantibodies were identified in the majority of AM and a-JDM patients ( $n=33 ; 84.1 \%$ and $n=12,80 \%$ respectively) (no results available for $7 \mathrm{AM}$ and 3 a-JDM patients). Patients with AM were characterised by a wide range of autoantibodies including anti-synthetase (anti-Jo-1 and anti-PI-12) antibodies ( $n=7)$; patients with autoimmune rheumatic disease overlap (anti-Ro, anti-PmSCL and anti-RNP) antibodies ( $n=9$ ); cancer associated (anti-NXP2 and anti-TIF1 $\gamma$ ) group ( $n=4)$, and severe skin (anti-Mi2) group $(n=3)$. Eleven patients had multiple autoantibodies detected, of which 8 were positive for anti-Ro in addition to a second antibody. Unfortunately, complete data were not available for the a-JDM cohort.

AM patients were treated with a broader spectrum of non-biologic drugs including tacrolimus, cyclosporine and intravenous immunoglobulin-G (IVIG) compared to aJDM patients. Rituximab was the only biologic used in the AM cohort ( $n=7,15.9 \%)$, whereas infliximab $(n=2,13.3 \%)$, adalimumab $(n=1,6.7 \%)$ and rituximab $(n=1,6.7 \%)$ were used in the a-JDM cohort.

Overall analysis of these IIM patient subgroups showed that patients with DM with cancer had elevated ESR $(p=0.03)$ and higher MITAX score (although this was not significant). No other significant differences were identified between groups in terms of clinical and disease features, including skin disease (Tables 1 and 2 and Supplementary Figure 1).

\section{Distinct immune signatures characterise adult and juvenile DM}

In order to assess whether the different patient groups were characterised by unique immune cell profiles in depth immune phenotyping was performed including analysis 
of 33 PBMC populations (Supplementary Figure 3 and Supplementary Table 2 for gating strategies) comprising T-cell, B-cell and monocyte sub-populations. Significant changes in immune profile between AM patients and age-matched healthy controls (Figure 1A and B, Supplementary Table 3) were identified.

Stratification of IIM patients revealed unique immune signatures associated with disease subgroups including increased Th17 and regulatory T cell (Treg) populations and decreased memory B-cells (early(e)BM5) in patients with ADM compared to adult healthy controls (AHC) (Figure 1C) and decreased CD27+ B-cells and CD8+ CM T-cells in APM patients (Figure 1D). Patients with a-JDM were characterised by significantly decreased naïve B-cells (BM2), total $\mathrm{CD}^{+}$and $\mathrm{CD}^{+}$central memory (CM) and natural killer (NK) cell populations and increased total CD4+ T-cells in aJDM patients compared to teenage healthy controls (THC) (Figure 1E). Furthermore, when ADM and a-JDM patient groups were compared directly, ADM patients retained their unique elevated Th17/Treg and decreased memory B-cells signature but also had reduced CD8+ naïve and intermediate monocyte populations compared to a-JDM patients (Figure 1F). Age did not influence the disease-associated profiles when adult and teenage healthy controls were compared (Figure 1G). Refer to Supplementary Table 3 for specific values of significant differences.

\section{Distinct immune signatures were associated with myositis associated and specific autoantibody profiles.}

Complex patterns of myositis associated and specific autoantibodies have been widely linked to IIM subgroups and distinct clinical features(21) (22). Correlation of the immune phenotype of AM patients with their autoantibody status revealed patients with anti-synthetase (anti-Jo-1 and anti-PI-12) antibodies ( $n=7)$ had decreased $\mathrm{CD}^{2} 7^{+} \mathrm{B}$-cells compared to $\mathrm{AHC}$ (Figure $2 \mathrm{~A}$ ) and patients with autoimmune rheumatic disease overlap (anti-Ro, anti-PmSCL and anti-RNP) antibodies $(n=9)$ demonstrated a T-cell signature with increased Th17 and decreased $\mathrm{CD} 8{ }^{+} \mathrm{CM}$ populations (Figure 2B). The cancer associated (anti-NXP2 and anti-TIF1 $\gamma)$ group $(\mathrm{n}=4)$ showed increased total monocyte, intermediate monocyte and plasmablast (BM3-4) B-cell populations, but decreased total T-cells (Figure 2C). There were no significant phenotypic changes when the severe skin (anti-Mi2) group 
$(n=3)$ was compared to AHC (Figure 2D), or in patients with multiple autoantibody profiles or patients negative for autoantibodies (data not shown) compared to healthy controls. Thus, stratifying according to autoantibody profile also revealed specific immune phenotype profiles, in particular in the anti-synthetase and overlap subgroups.

\section{Disease activity did not drive increased Th17 cell frequency in AM patients}

The AM cohort was further assessed according to disease activity. Four disease activity groups were identified using the clinicians decision on treatment criteria; active $(n=4)$, mildly active $(n=9)$, remission on-treatment $(n=12)$ and remission offtreatment $(n=5)$. The immune profile of AM patients in remission did have altered immune signatures; patients in remission off-treatment were characterised by increased non-classical monocytes and naive B-cell populations and decreased memory CD27+B-cells (Figure $2 \mathrm{E}$ ); while Th17 cells were elevated in patients in remission on-treatment (Figure $2 \mathrm{~F}$ ), potentially reflecting immune changes that occur in $A M$ that continue into remission and are no longer dampened by treatment. However, the immune profile of patients with active or mildly active disease was not significantly altered compared to AHCs $(n=25)$ (Figure $2 \mathrm{G}$ and $2 \mathrm{H}$ ).

\section{Increased Th17 cells were associated with an IL-6 signature in AM patients} Notably, IL-6 is detected at high levels in the serum and muscle of IIM patients and has been linked to active disease (23). Therefore peripheral blood immune cell activation (measured by CD69 expression) and IL-6 production (detected by intracellular staining) was assessed (Supplementary Figure 4 for gating strategies). AM but not a-JDM patients had a significant increase in both immune cell activation and IL-6 production compared with healthy individuals (Supplementary Figure 5 A-C and Figure $3 \mathrm{~A}-\mathrm{C}$ ). Moreover, when AM patient subgroups were considered the IL-6 signature was only seen in the ADM patients (Figure 3D\&E). Furthermore, a significant positive correlation was observed between Th17 expansion and T-cell activation (measured by CD69 expression) and IL- 6 production by memory B-cells in AM patients (Figure 3F). No relationship was observed between IL-6 and Th17 expression and MITAX score (Supplementary Figure 6). 
Thus unique immune signatures were identified in ADM, APM and a-JDM patient subgroups. All patients had decreased $\mathrm{CD} 8{ }^{+} \mathrm{CM}$ cell frequencies. ADM patients had a T-cell signature with an increase in total CD4+ $4^{+}$-cells and Th17 cells, as well as increased pan-immune cell IL- 6 production and immune cell activation. APM patients had a B-cell signature with a decreased memory B-cells and did not produce IL-6 (Figure 4). In contrast to ADM, a-JDM patients did not have a Th17/LL-6 signature and were associated with increased B-cell memory cell frequencies compared to THCs. 


\section{DISCUSSION}

This study is the first to identify novel immune cell signatures in an heterogenous group of patients with the very rare disease IIM; distinct immune-phenotypes were identified between disease subtypes, ages and disease activity. Specifically, ADM but not APM patients were associated with expansion of Th17 cells associated with elevated IL-6 expression, but all patient groups had reduced $\mathrm{CD} 8{ }^{+} \mathrm{CM} \mathrm{T}$-cell frequencies. Previous studies in peripheral blood have highlighted changes in total immune cell populations including monocytes, $T$ and $B$ lymphocytes, but have not correlated changes across a large number of different lymphocyte sub-populations (24).

Our aim was to report unique peripheral blood immune signatures that distinguished each subtype of AM and a-JDM. Our findings suggest that ADM is a more IL-6 and IL-17 driven disease compared to APM or a-JDM. Th17 T-cells selectively produce IL-17 over interferon (IFN)- $\gamma$ and depend on IL-6 amongst other cytokines for their differentiation. The Th17 pathway plays a critical role in the induction and maintenance of chronic inflammation and autoimmunity and Th17 cells have been found in the muscle tissue of IIM patients(25). IL-17 in conjunction with IL-1 and TNF- $\alpha$ increases the expression of MHC class I on muscle cells, relevant in the context of IIM as there is overexpression of MHC class I(26). The induction of IL-6 by IL-17 may also be involved in the dysfunctional repair of inflamed muscle(25).

IL-17 inhibitors have been considered to be potentially beneficial for use in IIM patients based on increased IL-17 expression in muscle tissue(25). Multiple biologic drugs targeting the IL-17 and IL-6 pathway are in clinical use $(27,28)$. The heterogeneity in IL-17 and IL-6 expression reported here implies that not all IIM patients would be responsive to such inhibition.

This study confirms the existence of an expanded Th17 population in the peripheral blood of IIM patients $(29,30)$. Based on the results presented here, it would be important to establish a correlation between the proportion of Th17 cells in the 
periphery and inflamed tissue at diagnosis and to monitor the change over time on treatment with disease progression.

Previously AM patient subtypes have been described based on autoantibody profiles (31), which was also reflected in this analysis. Myositis associated antibodies are detected in both DM and PM, but there is a wider range found in DM. The anti-Jo1 and anti-SRP autoantibodies are more common in PM. However, anti-Mi2, anti-TIF1 $\gamma$, and anti-NXP2 are rarely detected in PM but more commonly in DM and a-JDM (32). The AM cohort for this study displayed comparable prevalence of myositis associated antibodies to that previously reported. In particular, PM patients were only positive for anti-Jo1 and anti-SRP. Unfortunately, accurate data was not available for the a-JDM cohort.

$B$ cells have been detected within the inflamed muscle of AM and JDM patients, suggesting that B cells exert a pathological function on muscles (12, 33). A notable autoantibody profile can be detected in most IIM patients implying one function of $B$ cells in this group of diseases is the production of autoantibodies (34). These results provided an insight into the phenotype and function of B cells within the peripheral blood of both AM and a-JDM patients. The B cell compartment was dysregulated in the peripheral blood; the AM patients had a lower proportion of CD27- and BM5 early (IgD-CD38+) B cells compared to a-JDM and AHC groups, and the a-JDM group had an increased BM2 transitional $\left(\lg D^{\text {hi } C D 38^{\text {hi }}}\right)$ population. However, studies in SLE have identified that the memory B cell populations, both CD27+ and -, were expanded and immunosuppressive resistant (35). The decrease of the CD27+ and BM5 B cell populations was most evident in the AM patients expressing antisynthetase (anti-Jo-1 and anti-PI-12) autoantibodies and those that were classified as in remission off treatment. Interestingly Piper et al have previously detailed an indepth investigation of $B$ cell populations in JDM from pre- and on-treatment patients, they showed that the memory $B$ cell population was lowest in pre-treatment patients (16). The low memory B cell population identified in IIM may be a coincidental nonpathogenic finding which better reflects changes in related populations. Interestingly, there was an increased Plasmablast (BM3-4) population seen in the ADM patients 
with cancer with associated anti-TIF1 $\gamma$ and anti-NXP2 positivity. This maybe a signature associated with the underlying cancer or specific antibody production(36).

The limitations of this study were that we did not have access to treatment naive patient samples, and therefore it was not possible to delineate the effect of treatment on the disease course and immune signatures. However, it is difficult to recruit adult patients at diagnosis naïve of treatment as the disease is very rare and patients frequently experience delayed or wrong diagnosis with previous steroid use(37). It would be important for future studies to define any changes in the immune signature over the treatment course, and therefore would require longitudinal samples that were matched disease activity scores.

In conclusion, this study has identified IL-17 and IL-6 as potential therapeutic targets in ADM patients but also revealed complex patterns of immune cell expression in APM and a-JDM patients that provide a base for future investigations focused on understanding the disease pathogenesis and development of more personalised choice of biologic therapies for patients with IIMs. 


\section{REFERENCES:Bibliography}

1. Venalis $P$, Lundberg IE. Immune mechanisms in polymyositis and dermatomyositis and potential targets for therapy. Rheumatology. 2014;53(3):397405.

2. Lundberg IE. The role of cytokines, chemokines, and adhesion molecules in the pathogenesis of idiopathic inflammatory myopathies. Current rheumatology reports. 2000;2(3):216-24.

3. Salomonsson S, Lundberg IE. Cytokines in idiopathic inflammatory myopathies. Autoimmunity. 2006;39(3):177-90.

4. Ceribelli A, De Santis M, Isailovic N, Gershwin ME, Selmi C. The Immune Response and the Pathogenesis of Idiopathic Inflammatory Myositis: a Critical Review. Clin Rev Allergy Immunol. 2017;52(1):58-70.

5. Salajegheh M, Rakocevic G, Raju R, Shatunov A, Goldfarb LG, Dalakas MC. $T$ cell receptor profiling in muscle and blood lymphocytes in sporadic inclusion body myositis. Neurology. 2007;69(17):1672-9.

6. Hofbauer M, Wiesener S, Babbe H, Roers A, Wekerle H, Dornmair K, et al. Clonal tracking of autoaggressive $T$ cells in polymyositis by combining laser microdissection, single-cell PCR, and CDR3-spectratype analysis. Proceedings of the National Academy of Sciences of the United States of America. 2003;100(7):4090-5.

7. Reed AM, Ernste $F$. The inflammatory milieu in idiopathic inflammatory myositis. Current rheumatology reports. 2009;11(4):295-301.

8. Klein R, Rosenbach M, Kim EJ, Kim B, Werth VP, Dunham J. Tumor necrosis factor inhibitor-associated dermatomyositis. Archives of dermatology. 2010;146(7):780-4.

9. Banica L, Besliu A, Pistol G, Stavaru C, lonescu R, Forsea AM, et al. Quantification and molecular characterization of regulatory $T$ cells in connective tissue diseases. Autoimmunity. 2009;42(1):41-9.

10. Chevrel G, Page G, Granet C, Streichenberger N, Varennes A, Miossec P. Interleukin-17 increases the effects of IL-1 beta on muscle cells: arguments for the role of $\mathrm{T}$ cells in the pathogenesis of myositis. Journal of neuroimmunology. 2003;137(1-2):125-33.

11. Tournadre A, Porcherot M, Cherin P, Marie I, Hachulla E, Miossec P. Th1 and Th17 balance in inflammatory myopathies: interaction with dendritic cells and possible link with response to high-dose immunoglobulins. Cytokine. 2009;46(3):297301.

12. Greenberg SA, Bradshaw EM, Pinkus JL, Pinkus GS, Burleson T, Due B, et al. Plasma cells in muscle in inclusion body myositis and polymyositis. Neurology. 2005;65(11):1782-7.

13. Radke J, Koll R, Preusse C, Pehl D, Todorova K, Schonemann C, et al. Architectural B-cell organization in skeletal muscle identifies subtypes of dermatomyositis. Neurol Neuroimmunol Neuroinflamm. 2018;5(3):e451. 14. Deakin CT, Yasin SA, Simou S, Arnold KA, Tansley SL, Betteridge ZE, et al. Muscle biopsy in combination with myositis-specific autoantibodies aids prediction of outcomes in juvenile dermatomyositis. Arthritis \& rheumatology. 2016;11(68):280616. 
15. Baek A, Park HJ, Na SJ, Shim DS, Moon JS, Yang Y, et al. The expression of BAFF in the muscles of patients with dermatomyositis. Journal of neuroimmunology. 2012;249(1-2):96-100.

16. Piper CJM, Wilkinson MGL, Deakin CT, Otto GW, Dowle S, Duurland CL, et al. CD19(+)CD24(hi)CD38(hi) B Cells Are Expanded in Juvenile Dermatomyositis and Exhibit a Pro-Inflammatory Phenotype After Activation Through Toll-Like Receptor 7 and Interferon-alpha. Front Immunol. 2018;9:1372.

17. Bohan A, Peter JB. Polymyositis and dermatomyositis (first of two parts). The New England journal of medicine. 1975;292(7):344-7.

18. Sultan SM, Allen E, Oddis CV, Kiely P, Cooper RG, Lundberg IE, et al. Reliability and validity of the myositis disease activity assessment tool. Arthritis and rheumatism. 2008;58(11):3593-9.

19. Yee CS, Cresswell L, Farewell V, Rahman A, Teh LS, Griffiths B, et al. Numerical scoring for the BILAG-2004 index. Rheumatology. 2010;49(9):1665-9.

20. Rider LG, Koziol D, Giannini EH, Jain MS, Smith MR, Whitney-Mahoney K, et al. Validation of manual muscle testing and a subset of eight muscles for adult and juvenile idiopathic inflammatory myopathies. Arthritis care \& research. 2010;62(4):465-72.

21. Betteridge Z, McHugh N. Myositis-specific autoantibodies: an important tool to support diagnosis of myositis. Journal of Internal Medicine. 2016;280(1):8-23.

22. Deakin CT, Yasin SA, Simou S, Arnold KA, Tansley SL, Betteridge ZE, et al. Muscle Biopsy Findings in Combination With Myositis-Specific Autoantibodies Aid Prediction of Outcomes in Juvenile Dermatomyositis. Arthritis \& Rheumatology. 2016;68(11):2806-16.

23. Bilgic H, Ytterberg SR, Amin S, McNallan KT, Wilson JC, Koeuth T, et al. Interleukin-6 and type I interferon-regulated genes and chemokines mark disease activity in dermatomyositis. Arthritis and rheumatism. 2009;60(11):3436-46.

24. O'Gorman MR, Corrochano V, Roleck J, Donovan M, Pachman LM. Flow cytometric analyses of the lymphocyte subsets in peripheral blood of children with untreated active juvenile dermatomyositis. Clin Diagn Lab Immunol. 1995;2(2):205-8. 25. Tournadre A, Miossec P. Interleukin-17 in inflammatory myopathies. Current rheumatology reports. 2012;14(3):252-6.

26. Nagaraju K, Casciola-Rosen L, Lundberg I, Rawat R, Cutting S, Thapliyal R, et al. Activation of the endoplasmic reticulum stress response in autoimmune myositis: potential role in muscle fiber damage and dysfunction. Arthritis and rheumatism. 2005;52(6):1824-35.

27. Kondo M, Murakawa Y, Matsumura T, Matsumoto O, Taira M, Moriyama M, et al. A case of overlap syndrome successfully treated with tocilizumab: a hopeful treatment strategy for refractory dermatomyositis? Rheumatology.

2014;53(10):1907-8.

28. Miossec P. Update on interleukin-17: a role in the pathogenesis of inflammatory arthritis and implication for clinical practice. RMD Open. 2017;3(1):e000284.

29. Tang X, Tian X, Zhang Y, Wu W, Tian J, Rui K, et al. Correlation between the frequency of Th17 cell and the expression of microRNA-206 in patients with dermatomyositis. Clinical \& developmental immunology. 2013;2013:345347.

30. Espinosa-Ortega F, Gomez-Martin D, Santana-De Anda K, Romo-Tena J, Villasenor-Ovies P, Alcocer-Varela J. Quantitative T cell subsets profile in peripheral blood from patients with idiopathic inflammatory myopathies: tilting the balance 
towards proinflammatory and pro-apoptotic subsets. Clinical and experimental immunology. 2015;179(3):520-8.

31. Habers GE, Huber AM, Mamyrova G, Targoff IN, O'Hanlon TP, Adams S, et al. Brief Report: Association of Myositis Autoantibodies, Clinical Features, and Environmental Exposures at Illness Onset With Disease Course in Juvenile Myositis. Arthritis \& rheumatology. 2016;68(3):761-8.

32. Ghirardello A, Bassi N, Palma L, Borella E, Domeneghetti M, Punzi L, et al. Autoantibodies in polymyositis and dermatomyositis. Current rheumatology reports. 2013;15(6):335.

33. Shah M, Mamyrova G, Targoff IN, Huber AM, Malley JD, Rice MM, et al. The clinical phenotypes of the juvenile idiopathic inflammatory myopathies. Medicine. 2013;92(1):25-41.

34. Nistala K, Wedderburn LR. Update in juvenile myositis. Current opinion in rheumatology. 2013;25(6):742-6.

35. Dorner T, Giesecke C, Lipsky PE. Mechanisms of B cell autoimmunity in SLE. Arthritis research \& therapy. 2011;13(5):243.

36. Lundgren S, Berntsson J, Nodin B, Micke P, Jirstrom K. Prognostic impact of tumour-associated B cells and plasma cells in epithelial ovarian cancer. J Ovarian Res. 2016;9:21.

37. Da Silva DM, Patel B, Werth VP. Dermatomyositis: A diagnostic dilemma. J Am Acad Dermatol. 2018;79(2):371-3.

Contributors: Design of research study; JJM, ECJ, MGLIW: Conducting experiments; MGLIW: Acquiring experimental data; MGLIW, CW, JJM, DAI: Analyzing data, MW, ECJ, JJM: Patient recruitment and assessment; JJM, DAI, CW, AR, MGLIW, JI: Acquisition of clinical data; JJM, DAI, CW, AR, MGLIW, JI; Writing the manuscript; MW, ECJ, JJM: Review of the manuscript; All authors: DAI, ECJ, JJM contributed equally as authors.

Funding: This work was supported by a grant from the Adolescent Centre at UCL UCLH and GOSH is funded by Arthritis Research UK (20164 and 21953), Great Ormond Street Children's Charity and the NIHR Biomedical Research Centres at GOSH and UCLH, the UCL Rheumatology Research Fund and an equipment grant from University College London Hospitals Biomedical Research Centre. The views expressed are those of the authors and not necessarily those of the NHS, the NIHR or the Department of Health.

Competing interests: The authors have declared that no conflict of interest exists.

Ethics approval: North Harrow ethics committee approval (REC 11/0101). 


\section{Acknowledgements:}

We would like to thank all patients and healthy volunteers for donating to the study. Special thanks to all the team that work with the ARUK Centre for Adolescent Rheumatology. We would also like to thank Dr J. Evans, Dr. A. Eddaoudi and staff from the UCL flow cytometry facility units for their assistance in flow cytometry. Furthermore, we would like to thank lab members for patient recruitment and sample processing. 
Table 1:

\begin{tabular}{|c|c|c|c|c|c|c|c|c|c|}
\hline & Patients & & & & & & & Controls & \\
\hline $\begin{array}{c}\text { Patient } \\
\text { characteristics }\end{array}$ & $\begin{array}{c}\text { Adult } \\
\text { Myositis } \\
\text { samples } \\
(n=44)\end{array}$ & & & & & & $\begin{array}{c}\text { Juvenile DM } \\
\text { (adolescent } \\
\text { onset) samples } \\
(n=15)\end{array}$ & $\begin{array}{l}\text { Adult healthy } \\
\text { control } \\
\text { samples } \\
(n=25)\end{array}$ & $\begin{array}{c}\text { Adolescent } \\
\text { healthy } \\
\text { control } \\
\text { samples } \\
(n=15)\end{array}$ \\
\hline $\begin{array}{l}\text { Sub-types of } \\
\text { myositis }\end{array}$ & All & Dermatomyositis & Polymyositis & $\begin{array}{l}\text { Dermatomyositis } \\
\text { with cancer }\end{array}$ & $\begin{array}{l}\text { Dermatomyositis } \\
\text { with overlap } \\
\text { syndrome }\end{array}$ & $\begin{array}{l}\text { Polymyositis } \\
\text { with overlap } \\
\text { syndrome }\end{array}$ & All & All & All \\
\hline Number of patients & 44 & 19 & 9 & 4 & 7 & 5 & 15 & 25 & 15 \\
\hline $\begin{array}{l}\text { Age at diagnosis } \\
\text { (years), median } \\
\text { [IQR] }(p=0.0632)\end{array}$ & $\begin{array}{l}39.82 \\
{[31.65-} \\
49.00]\end{array}$ & $\begin{array}{l}34.02 \text { [30.33- } \\
48.96]\end{array}$ & $\begin{array}{l}41.29[34.10- \\
44.07]\end{array}$ & 47.83 [27.65-60.62] & 39.87 [31.30-45.80] & $\begin{array}{l}39.64 \text { [32.47- } \\
43.04]\end{array}$ & $\begin{array}{l}14.78[14.02- \\
16.01]\end{array}$ & & \\
\hline $\begin{array}{l}\text { Age at sample } \\
\text { (years), median } \\
\text { [IQR] }(p=0.2194)\end{array}$ & $\begin{array}{l}55.48 \\
{[47.59-} \\
60.33]\end{array}$ & $\begin{array}{l}55.80[46.21- \\
59.99]\end{array}$ & $\begin{array}{l}52.37[48.35- \\
53.10]\end{array}$ & 63.04 [59.81-67.99] & $49.20[46.82-53.47]$ & $\begin{array}{l}59.72[55.96- \\
66.73]\end{array}$ & $\begin{array}{l}21.48[19.07- \\
23.19]\end{array}$ & $\begin{array}{l}51.26[43.23- \\
58.31]\end{array}$ & $\begin{array}{l}20.11[17.40- \\
22.28]\end{array}$ \\
\hline $\begin{array}{l}\text { Time since } \\
\text { diagnosis (years), } \\
\text { median [IQR] } \\
(\mathrm{p}=0.5453)\end{array}$ & $\begin{array}{l}9.64[3.39- \\
23.13]\end{array}$ & 12.39 [4.61-22.29] & $\begin{array}{l}6.43[5.15- \\
10.97]\end{array}$ & $0.34[0-6.23]$ & $11.02[1.02-24.86]$ & $\begin{array}{l}23.48[7.98- \\
23.69]\end{array}$ & 6.09 [5.28-7.82] & & \\
\hline $\begin{array}{l}\text { Sex, (F/M) } \\
{[\mathrm{p}=0.3013]}\end{array}$ & $34 / 10$ & $12 / 7$ & $9 / 0$ & $4 / 0$ & $5 / 2$ & $4 / 1$ & $11 / 4$ & $14 / 11$ & $11 / 4$ \\
\hline \multicolumn{10}{|l|}{$\begin{array}{l}\text { Ethnicty, n (\%) } \\
{[0.0968]}\end{array}$} \\
\hline White British & $17(38.64)$ & $10(52.63)$ & $1(11.11)$ & $2(50.00)$ & $3(42.86)$ & $1(20)$ & $9(60.00)$ & $14(56.00)$ & $6(40.00)$ \\
\hline Black Carribean & $9(20.45)$ & $1(5.26)$ & $5(55.56)$ & $1(25)$ & $1(14.29)$ & $1(20)$ & $2(13.33)$ & $0(0.00)$ & $0(0.00)$ \\
\hline Black African & $3(6.81)$ & $1(5.26)$ & $0(0.00)$ & $0(0.00)$ & $1(14.29)$ & $1(20)$ & $1(6.67)$ & $0(0.00)$ & $1(6.67)$ \\
\hline South Asian & $7(15.91)$ & $2(10.53)$ & $1(11.11)$ & $0(0.00)$ & $2(28.57)$ & $2(40.00)$ & $0(0.00)$ & $2(8.00)$ & 5 (33.33) \\
\hline East Asian & $0(0.00)$ & $0(0.00)$ & $0(0.00)$ & $0(0.00)$ & $0(0.00)$ & $0(0.00)$ & $0(0.00)$ & $0(0.00)$ & $1(6.67)$ \\
\hline White other & 7 (15.91) & $4(21.05)$ & 2 (22.22) & $1(25)$ & $0(0.00)$ & $0(0.00)$ & $0(0.00)$ & $7(28.00)$ & $0(0.00)$ \\
\hline South American & $0(0.00)$ & $0(0.00)$ & $0(0.00)$ & $0(0.00)$ & $0(0.00)$ & $0(0.00)$ & $0(0.00)$ & $0(0.00)$ & $0(0.00)$ \\
\hline Other & $1(2.27)$ & $1(5.26)$ & $0(0.00)$ & $0(0.00)$ & $0(0.00)$ & $0(0.00)$ & $3(20.00)$ & $0(0.00)$ & $1(6.67)$ \\
\hline Unknown & $0(0.00)$ & $0(0.00)$ & $0(0.00)$ & $0(0.00)$ & $0(0.00)$ & $0(0.00)$ & $0(0.00)$ & $2(8.00)$ & $0(0.00)$ \\
\hline
\end{tabular}


Table 2:

\begin{tabular}{|c|c|c|c|c|c|c|c|}
\hline Patient characteristics & $\begin{array}{l}\text { Adult Myositis } \\
\text { samples }(n=44)\end{array}$ & & & & & & $\begin{array}{c}\text { Juvenile DM } \\
\text { (adolescent onset) } \\
\text { samples ( } n=15) \\
\end{array}$ \\
\hline Sub-types of myositis & All & $\begin{array}{c}\text { Dermatomyositis } \\
(n=19)\end{array}$ & Polymyositis $(n=9)$ & $\begin{array}{l}\text { Dermatomyositis } \\
\text { with cancer }(n=4)\end{array}$ & $\begin{array}{l}\text { Dermatomyositis with } \\
\text { overlap syndrome } \\
(n=7)\end{array}$ & $\begin{array}{l}\text { Polymyositis with } \\
\text { overlap syndrome } \\
\qquad(n=5)\end{array}$ & All \\
\hline $\begin{array}{l}\text { ESR mm/hour } \\
{[p=0.0394]}\end{array}$ & $18.5[8.00-28.25]$ & $9.00[5.75-17.50]$ & $25.00[22.5-30.00]$ & $40.00[33.00-43.50]$ & 23.00 [16.50-37.50] & $24.5[16.25-36.25]$ & $16.5[7.50-22.50]$ \\
\hline CRP mg/L $[p=0.7019]$ & $2[0.70-5.80]$ & $1.60[0.78-2.65]$ & $2.3[0.70-6.48]$ & $3.00[1.88-28.45]$ & $4.45[1.73-17.90]$ & $4.90[1.65-10.05]$ & $2.75[2.23-5.00]$ \\
\hline CK U/L $[p=0.6966]$ & $147[93-364]$ & $195[125-301]$ & $230.5[142-308]$ & 116 [107.75-696.50] & 100 [92.25-949.25] & 87 [75.5-192.25] & 106 [76.50-464.75] \\
\hline MITAX score $(p=0.063)$ & $1.50[0.00-9.00]$ & $1.00[0.00-9.00]$ & $3.00[1.00-9.00]$ & $9.50[1.75-19.00]$ & $2.00[0.50-10.50]$ & $0.00[0.00-2.25]$ & $0.00[0.00-4.00]$ \\
\hline MITAX skin score & $0[0-12]$ & & & & & & $0[0-8]$ \\
\hline MMT8 $(0-80)$ & Not done & Not done & Not done & Not done & Not done & Not done & $80[77-80]$ \\
\hline \multicolumn{8}{|l|}{$\begin{array}{l}\text { Myositis-specific } \\
\text { autoantibodies, } n(\%) \\
{[p=0.3022]}\end{array}$} \\
\hline Anti-Jo-1 & $6(13.63)$ & $1(5.26)$ & $4(44.44)$ & $0(0.00)$ & $1(14.29)$ & $0(0.00)$ & $0(0.00)$ \\
\hline Anti-Ro & $3(6.81)$ & $2(10.53)$ & $0(0.00)$ & $1(25)$ & $0(0.00)$ & $0(0.00)$ & $1(13.33)$ \\
\hline Anti-PL12 & $1(2.27)$ & $1(5.26)$ & $0(0.00)$ & $0(0.00)$ & $0(0.00)$ & $0(0.00)$ & $0(0.00)$ \\
\hline Anti-PM-Scl75 & $2(4.54)$ & $1(5.26)$ & $0(0.00)$ & $0(0.00)$ & $0(0.00)$ & $1(20)$ & $0(0.00)$ \\
\hline Anti-Mi2 & $3(6.81)$ & $3(15.78)$ & $0(0.00)$ & $0(0.00)$ & $0(0.00)$ & $0(0.00)$ & $1(6.67)$ \\
\hline Anti-Smith & $1(2.27)$ & $1(5.26)$ & $0(0.00)$ & $0(0.00)$ & $0(0.00)$ & $0(0.00)$ & $0(0.00)$ \\
\hline Anti-SRP & $1(2.27)$ & $0(0.00)$ & $1(11.11)$ & $0(0.00)$ & $0(0.00)$ & $0(0.00)$ & $0(0.00)$ \\
\hline Anti-NXP2 & $1(2.27)$ & $1(5.26)$ & $0(0.00)$ & $0(0.00)$ & $0(0.00)$ & $0(0.00)$ & $0(0.00)$ \\
\hline TIF1-gamma & $3(6.81)$ & $2(10.53)$ & $0(0.00)$ & $1(25)$ & $0(0.00)$ & $0(0.00)$ & $0(0.00)$ \\
\hline Multiple & $9(20.45)$ & $2(10.53)$ & $0(0.00)$ & $0(0.00)$ & $4(57.14)$ & $3(60)$ & $5(33.33)$ \\
\hline Negative & $7(15.90)$ & $3(15.78)$ & $3(33.33)$ & $1(25)$ & $0(0.00)$ & $0(0.00)$ & $0(0.00)$ \\
\hline Not done & $7(15.90)$ & $2(10.53)$ & $1(11.11)$ & $1(25)$ & $2(28.57)$ & $1(20)$ & $3(20.00)$ \\
\hline \multicolumn{8}{|l|}{$\begin{array}{l}\text { Medications (at time of } \\
\text { sample) } n(\%): \\
{[p=0.3237]}\end{array}$} \\
\hline Prednisolone & $28(63.63)$ & $10(52.63)$ & $4(44.44)$ & $3(75)$ & $7(100)$ & $4(80)$ & $8(53.33)$ \\
\hline Methotrexate & $5(11.36)$ & $2(10.53)$ & $2(22.22)$ & $0(0.00)$ & $1(14.29)$ & $0(0.00)$ & $10(66.67)$ \\
\hline Azathioprine & $9(20.45)$ & $2(10.53)$ & $2(22.22)$ & $1(25.00)$ & $4(57.14)$ & $0(0.00)$ & $2(13.33)$ \\
\hline Rituximab past & $7(15.91)$ & $2(10.53)$ & $2(22.22)$ & $0(0.00)$ & $1(14.29)$ & $2(40.00)$ & $1(6.67)$ \\
\hline Hydroxychloriquine & $5(11.36)$ & $3(15.78)$ & $1(11.11)$ & $0(0.00)$ & $1(14.29)$ & $0(0.00)$ & $6(40.00)$ \\
\hline MMF & $8(18.18)$ & $5(26.32)$ & $1(11.11)$ & $0(0.00)$ & $1(14.29)$ & $1(20)$ & $2(13.33)$ \\
\hline Tacrolimus & $3(6.812)$ & $1(5.26)$ & $1(11.11)$ & $0(0.00)$ & $1(14.29)$ & $0(0.00)$ & $0(0.00)$ \\
\hline Cyclosporine & $2(4.54)$ & $2(10.53)$ & $0(0.00)$ & $0(0.00)$ & $0(0.00)$ & $0(0.00)$ & $0(0.00)$ \\
\hline IVIG & $3(6.82)$ & $2(10.53)$ & $0(0.00)$ & $1(25)$ & $0(0.00)$ & $0(0.00)$ & $0(0.00)$ \\
\hline Infliximab & $0(0.00)$ & $0(0.00)$ & $0(0.00)$ & $0(0.00)$ & $0(0.00)$ & $0(0.00)$ & $2(13.33)$ \\
\hline
\end{tabular}




\section{FIGURE LEGENDS}

Table 1: Demographic features of the adult myositis, adolescent-onset-JDM, adult and teenage healthy control cohorts at time of sample. 44 adult myositis (AM) patients and 15 adolescent onset-juvenile dermatomyositis (a-JDM) patients (adolescence defined as 10-19 years of age) were recruited. The AM group was divided into subgroups; dermatomyositis (ADM), polymyositis (APM), dermatomyositis with cancer, dermatomyositis with overlap and polymyositis with overlap.

Table 2: Clinical and serological features of the adult myositis, adolescentonset-JDM, adult and teenage healthy control cohorts at time of sample. 44 adult myositis (AM) patients and 15 adolescent onset-(a)-juvenile dermatomyositis (a-JDM) patients (adolescence defined as 10-19 years of age) were recruited. The AM group was divided into subgroups; dermatomyositis (ADM), polymyositis (APM), dermatomyositis with cancer, dermatomyositis with overlap and polymyositis with overlap. For each group, the clinical and serological data were recorded at time of PBMC sample including; erythrocyte sedimentation rate (ESR) [normal range $<20 \mathrm{~mm} /$ hour], C-reactive protein (CRP) [normal range $<5 \mathrm{mg} / \mathrm{L}$ ], creatine kinase (CK) [normal range <150U/L], MITAX score, MITAX skin score (median with range) MMT8 score [normal range $>78$ ], auto-antibodies and medication. The binary measures were analysed by Chi-square test and the continuous variables were analysed by one-way ANOVA. $p \leq 0.05$ for significance. 

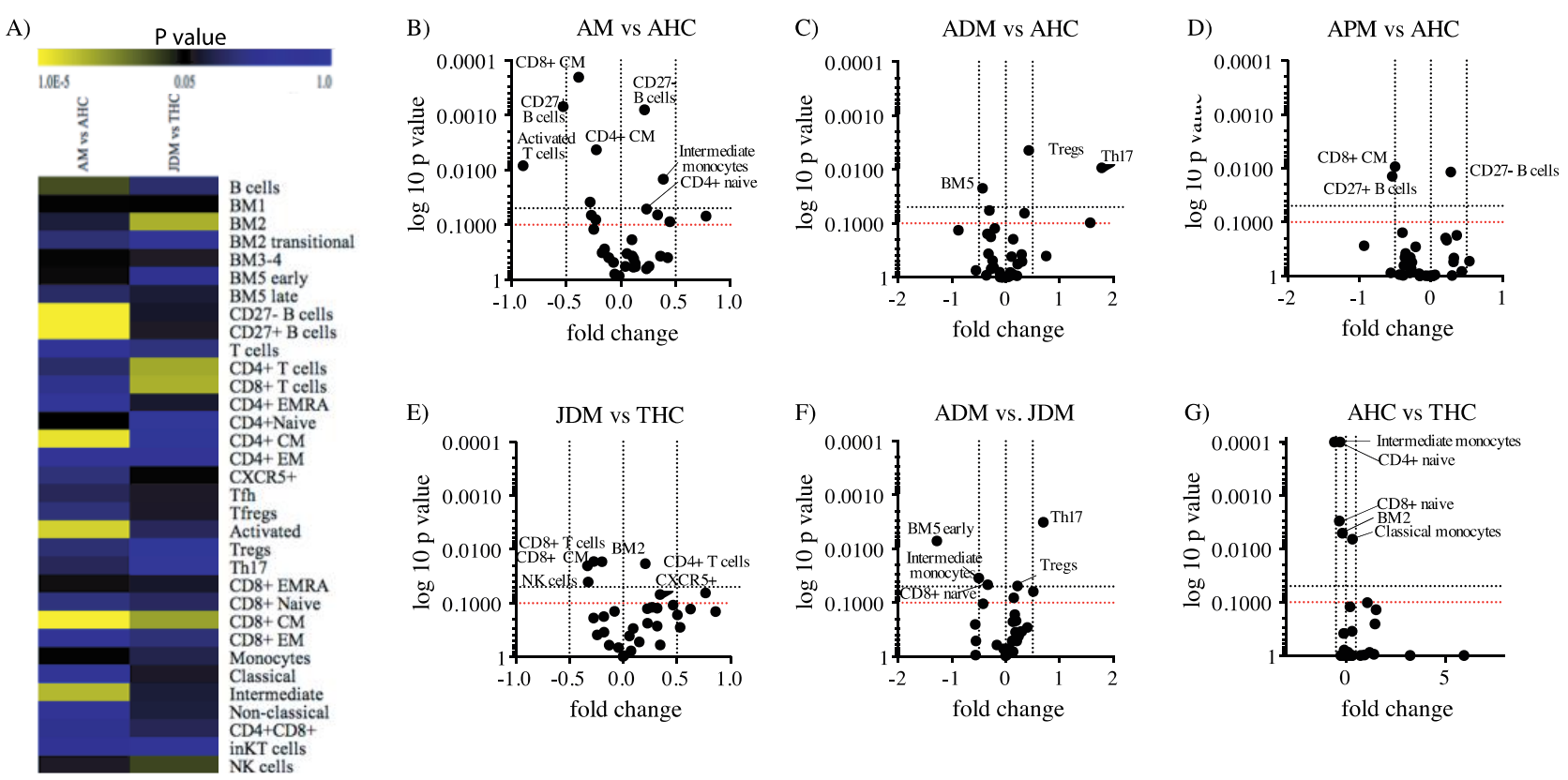

Figure 1: Heterogeneous immune cell profiles in IIM patient subgroups. The frequency of 33 PBMC sub-populations were assessed by multiparameter flow cytometry from 44 adult myositis (AM) patients, 25 adult healthy controls (AHC), 15 adolescent-onset juvenile dermatomyositis (a-JDM) patients and 15 teenage healthy controls (THC). (A) Heat map demonstrating the statistical difference between each cell population by comparing the mean of each group; AM vs. AHC and a-JDM vs. THC. A student t-test calculated the $p$-values. Black to yellow $-p<0.05$. Blue to black $-p>0.05$. Volcano plots are 2-D scatter plots that visualise significance, direction and the magnitude of changes within a given comparison. Volcano plots showing log2 scale fold change ( $x$-axis) and $\log 10$ scale $p$ value ( $y$-value) for each cell population when sample groups were compared. A negative fold change indicates that the cell population for the first named patient group was lower than the second named patient or healthy control group. A positive fold change indicates that the cell population for the first named patient group was higher than the second named patient or healthy control group. B) AM) vs. AHC), C) adult dermatomyositis (ADM) $(n=19)$ vs. $A H C), D)$ adult polymyositis (APM) ( $n=9)$ vs. AHC, E) a-JDM vs. THC, F) ADM vs. a-JDM G) AHC vs. THC. P values were calculated by one-way ANOVA. All $p$-values represent adjusted $p$-values calculated by Tukey's multiple comparison. Stringent significance $p \leq 0.05$ (horizontal black dotted line). Adjusted significance by false discovery rate (FDR) for small sample size $p \leq 0.1$ (horizontal red dotted line). The fold change significance is $\leq-0.5$ and $\geq 0.5$ (vertical dotted black lines). 

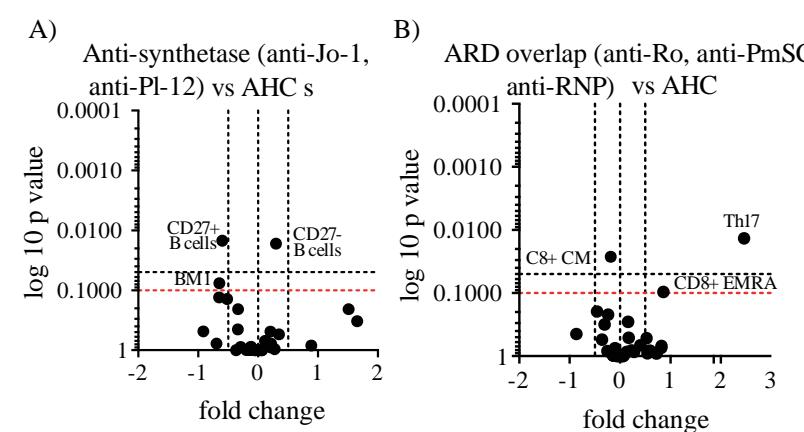

fold change
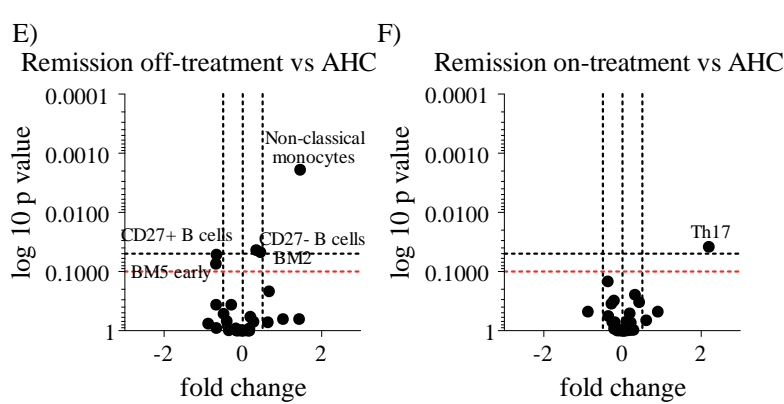
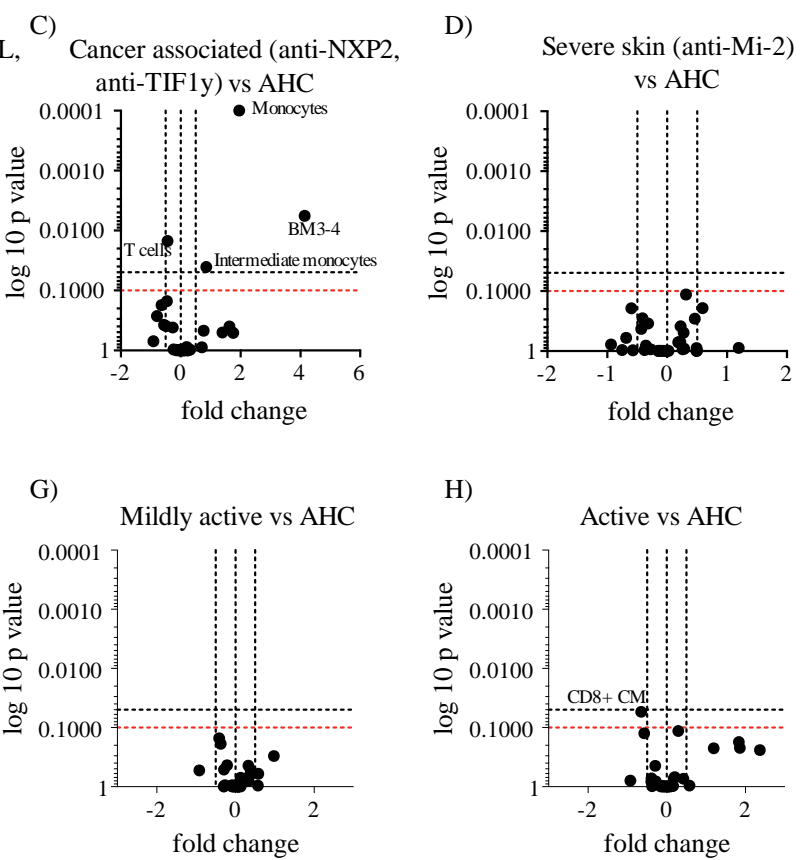

Figure 2: Heterogeneous immune cell profiles in IIM patient subgroups: autoantibody and disease activity. Volcano plots showing fold change (x-axis) and $p$ value ( $Y$-axis) for each cell population when $A M$ autoantibody groups were compared with AHC samples. A) Anti-synthetase group (anti-Jo-1 and anti-PI-12) $(n=7)$ compared to AHC ( $n=25)$. B) Autoimmune rheumatic disease overlap (ARD) group (anti-Ro, anti-PmScl and anti-RNP) ( $n=9)$ compared to AHC. C) Cancer associated group (anti-NXP2 and anti-TIF1 $\gamma)(n=4)$ compared to AHC. D) Severe skin group (anti-Mi2) $(n=3)$ compared to AHC. Volcano plots represent the fold change and $p$ value for each cell population when AM disease activity groups were compared. Patients' with cancer, pregnant, treated with rituximab or diagnosed in childhood were removed from this analysis. E): Remission off-treatment $(n=5)$ compared to $\mathrm{AHC}(\mathrm{n}=25)$. F) Remission on-treatment $(\mathrm{n}=12)$ compared to $\mathrm{AHC}$ $(n=25)$. G) Mildly active $(n=9)$ compared to $A H C(n=25)$. H) Active $(n=4)$ compared to AHC ( $n=25)$. $P$ values were calculated by one-way ANOVA. All $p$-values represent adjusted $p$-values calculated by Tukey's multiple comparison. Stringent significance $p \leq 0.05$ (horizontal black dotted line). Adjusted significance by false discovery rate (FDR) for small sample size $p \leq 0.1$ (horizontal red dotted line). The fold change significance is $\leq-0.5$ and $\geq 0.5$ (vertical dotted black lines). 
A)

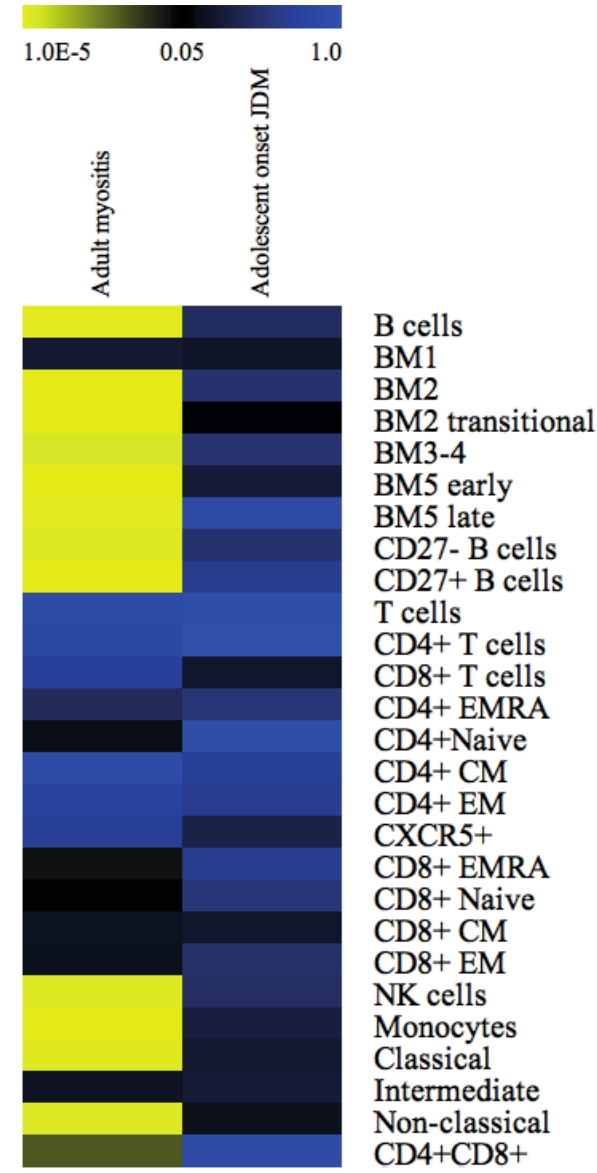

B)

IL-6 AM vs AHC

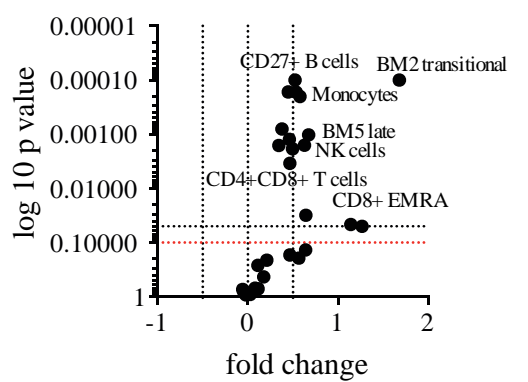

D)

IL-6 ADM vs AHC

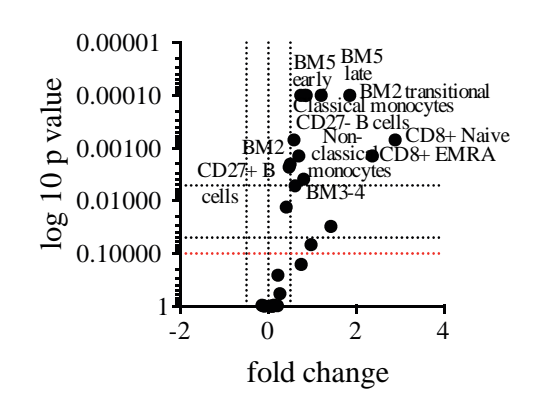

F) Th17 correlated with IL-6 and CD69 expression
IL-6 JDM vs THC

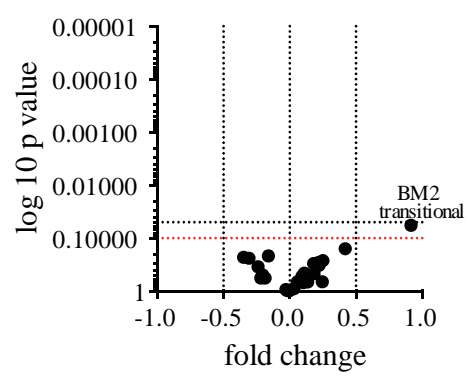

E)
IL-6 APM vs AHC
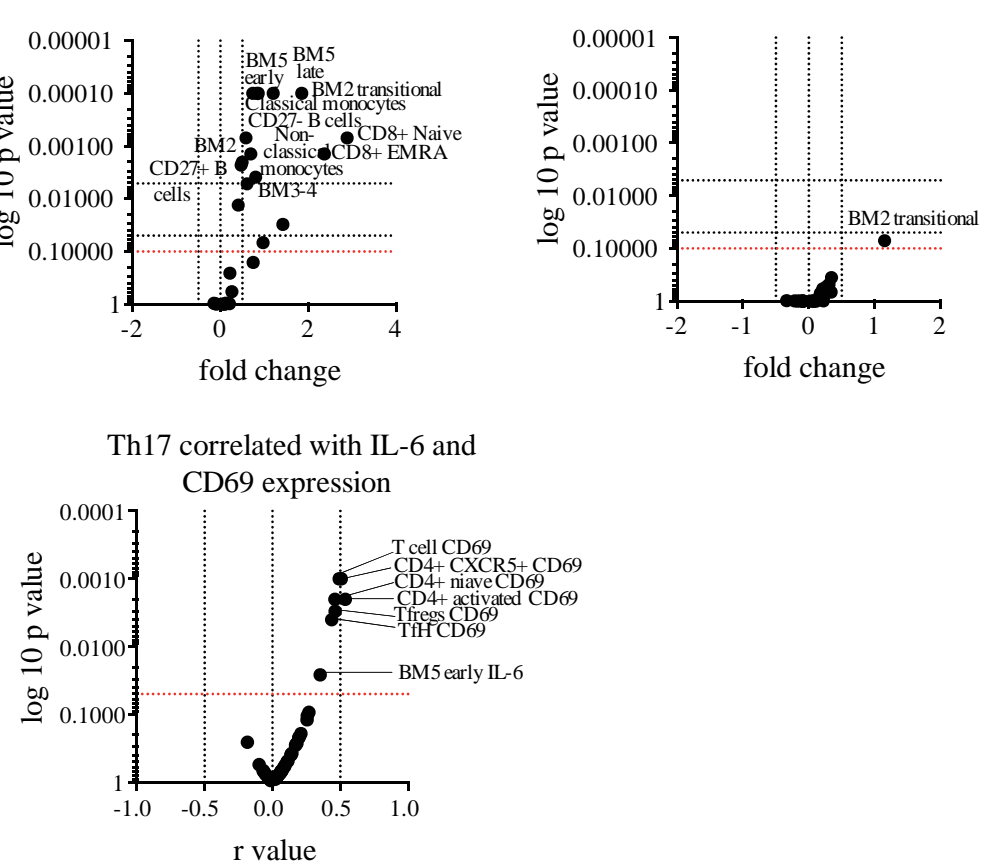

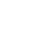

Figure 3: Significant differences in cell populations expression of IL-6 and the Th17 population, a comparison of IIM to healthy samples. IL-6 expression in 27 PBMC subpopulations was assessed by flow cytometry from 44 adult myositis (AM) patients, 25 adult healthy controls (AHC), 15 adolescent-onset juvenile dermatomyositis (a-JDM) patients and 15 teenage healthy controls (THC). A) Heat map showing the statistical difference between each cell population by comparing the mean of each group; AM vs. AHC, and a-JDM vs. THC. A student t-test calculated the $p$-values. Black to yellow - $p \leq 0.05$. Blue to black - $p \geq 0.05$. Volcano plots identifying significant differences and fold change of IL-6 expression in immune cell populations. B) AM $(n=44)$ compared to AHC ( $n=25)$. C) a-JDM $(n=15)$ compared to THC $(n=15)$. D) ADM $(n=19)$ compared to AHC $(n=25)$. E) APM $(n=9)$ compared to AHC ( $n=25)$. P values were calculated by multiple t-test, significance $p \leq$ 0.05 (bottom horizontal dotted black line). Multiple comparison was calculated by the Holm-Sidak method, therefore adjusted $p$ value significance $p \leq 0.005116$ (top 
horizontal dotted black line). Adjusted significance by FDR for small sample size $p \leq 0.1$ (red dotted line). The fold change significance is $\leq-0.5$ and $\geq 0.5$ (vertical dotted black lines). F) A volcano plot showing all PBMC populations, from the total AM group, expression of CD69 and IL-6 correlation to Th17 frequency. Pearson's correlation was conducted, positive $r$ value represents a positive correlation and negative $r$ value represents a negative correlation. Significance reached $p \leq 0.05$ (horizontal dotted red line). 


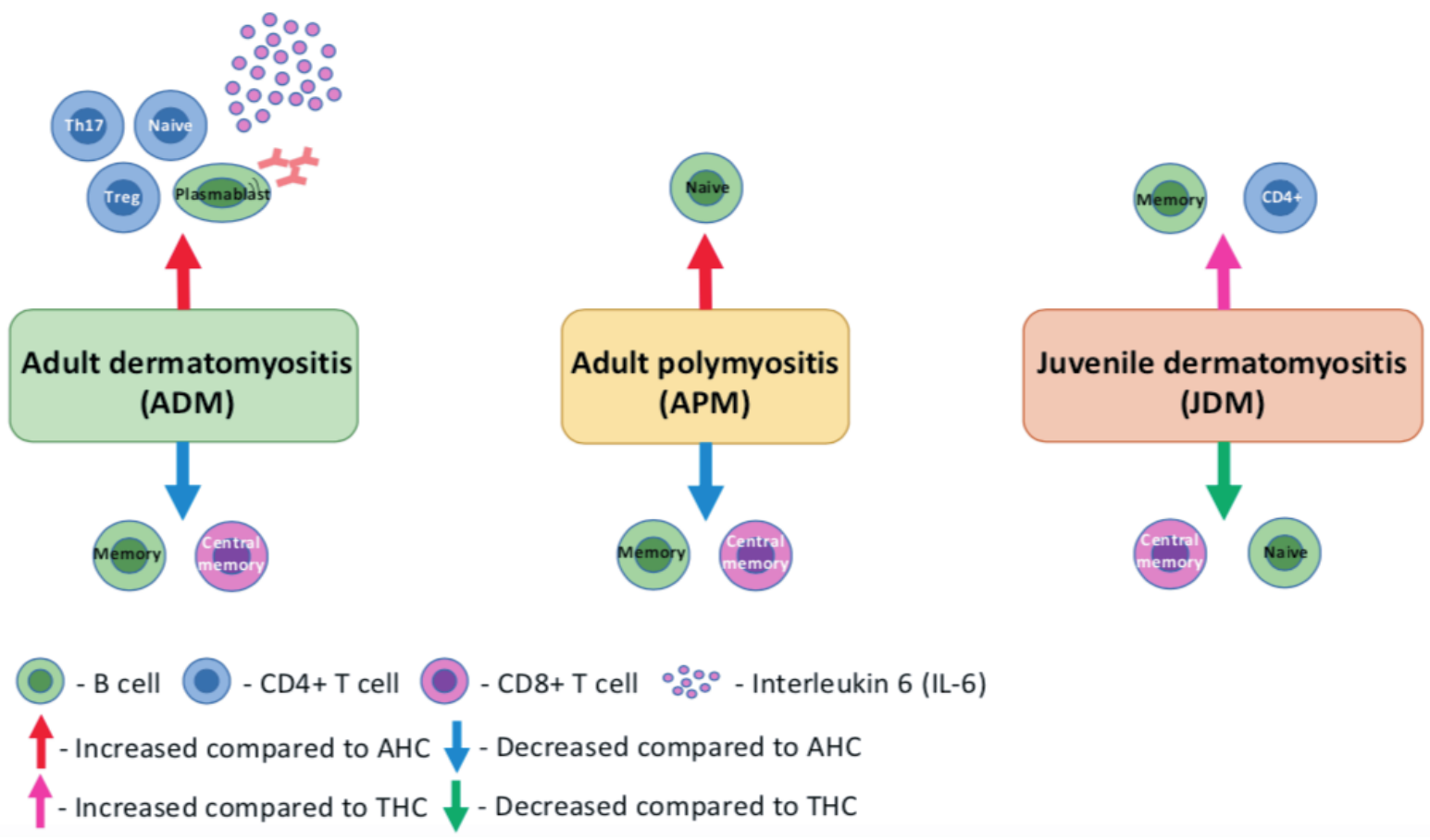

Figure 4: Different immune signatures in adult dermatomyositis, polymyositis and juvenile dermatomyositis. Different immune signatures in adult dermatomyositis and polymyositis compared to adult healthy control and adolescentonset juvenile dermatomyositis compared to teenage healthy control. ADM was characterised by an increase of $\mathrm{CD} 4^{+}$T-cells including; Th17, naïve T-cells and Tregs, but also plasmablasts in cancer associated dermatomyositis and an increased cellular expression of IL-6. Common to both ADM and APM there was a decrease of memory B-cells and central memory $\mathrm{CD} 8^{+} \mathrm{T}$-cells. This decrease in central memory CD8 ${ }^{+}$T-cells was also noted in a-JDM. 